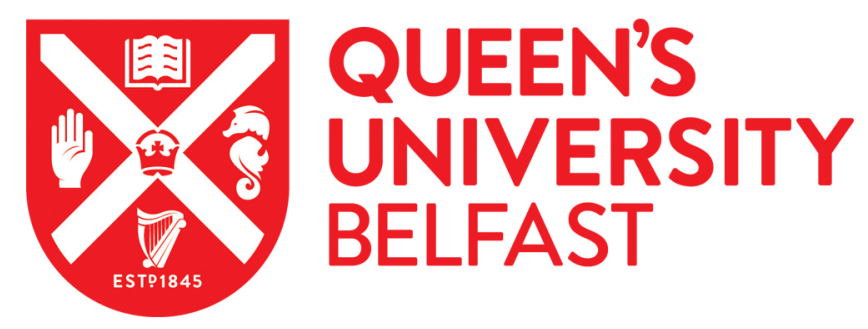

\title{
A global analysis of enemy release and its variation with latitude
}

Xu, M., Mu, X., Zhang, S., Dick, J. T. A., Zhu, B., Gu, D., Yang, Y., Luo, D., \& Hu, Y. (2021). A global analysis of enemy release and its variation with latitude. Global Ecology and Biogeography, 30(1), 277-288. https://doi.org/10.1111/geb.13229

\section{Published in:}

Global Ecology and Biogeography

\section{Document Version:}

Peer reviewed version

\section{Queen's University Belfast - Research Portal:}

Link to publication record in Queen's University Belfast Research Portal

\section{Publisher rights}

Copyright 2021 Wiley. This work is made available online in accordance with the publisher's policies. Please refer to any applicable terms of use of the publisher.

\section{General rights}

Copyright for the publications made accessible via the Queen's University Belfast Research Portal is retained by the author(s) and / or other copyright owners and it is a condition of accessing these publications that users recognise and abide by the legal requirements associated with these rights.

Take down policy

The Research Portal is Queen's institutional repository that provides access to Queen's research output. Every effort has been made to ensure that content in the Research Portal does not infringe any person's rights, or applicable UK laws. If you discover content in the Research Portal that you believe breaches copyright or violates any law, please contact openaccess@qub.ac.uk. 


\section{Abstract}

2 Aim: The enemy release hypothesis (ERH) posits that exotic species suffer lower enemy damage than natives, and thus promotes their successful invasion. However, the

4 generality of lower damage for exotics remains widely debated. A recent view proposes

5 that the enemy release (ER) effect could systematically change with latitude, potentially

6 helping explain these inconsistencies. Here, we test whether exotic plant species suffer

7 consistently lower herbivore damage relative to natives, and whether ER varies with

8 latitude.

9 Location: Global.

10 Time period: $1960-2018$.

11 Major taxa studied: Plants.

12 Methods: Using leaf herbivory data for 1,098 plant species, we compared the herbivory

13 rate between exotic and native plants across all taxa, in the introduced and native range,

14 or and between exotic and native species co-occurring in the same community. Then,

15 we tested the interaction effect between origin (exotic vs. native) and latitude to assess

16 the-variation in

17 its interaction with latitude changed with plant growth forms (woody vs. non-woody).

18 Finally, based on two ER effect-size datasets, two meta-regressions were performed to

19 demonstrate the relationship between ER and latitude.

20 Results: Leaf herbivory rates were commonly lower for exotics than natives. However, 21 thise differential herbivory rate between exoties and natives-was only significant for 
22 woody plants. No significant interactions were foundexisted between origin and

23 latitude, indicating that ER did not change with latitude. The meta-regressions also

24 demonstrated that ER was not significantly correlated with latitude.

25 Main conclusions: The widespread lower herbivory rate for exotic compared tothan

26 native woody plant species supports the ERH for exotic woody plants. Invariable

27 Consistent ER with latitude indicates that ERit should play a similar role regardless of

28 latitude. One future challenge is to assess to what extent ER in woody exotic plants

29 translates to performance advantages and hence influences their invasion success.

\section{KEYWORDS}

31 enemy release hypothesis, latitudinal gradient, growth forms, herbivory rate, exotic

32 species, native species, biological invasions, phylogenetic correlation, spatial

33 autocorrelation 
Biological invasions have led to significant ecological, social, and economic consequences, and understanding why particular exotic species successfully invade, as well as why some communities are more easily invaded, has become a major challenge in the field of invasion ecology (Kolar \& Lodge, 2001; Mack et al., 2000). An intuitively attractive and popular explanation is the enemy release hypothesis (ERH), which posits that ${ }_{2}$ in the introduced range, exotic species experience a decrease in enemy damage, resulting in a high abundance and wide distribution (Elton, 1958; Keane \& Crawley, 2002; Mitchell \& Power, 2003). Enemy release (ER) has also been viewed as an important mechanism explaining the ecological impacts of exotic species (Ricciardi, Hoopes, Marchetti, \& Lockwood, 2013), as well as a justification for the introduction of natural enemies in order to control them (Hajek \& Eilenberg, 2018).

Despite its central importance in understanding the causes of invasion a-successfut 47 invasion, the ERH remains widely debated (Colautti, Ricciardi, Grigorovich, \& MacIsaac, 2004; Maron \& Vila, 2001; Parker \& Gilbert, 2007). For animal-parasite and plant-pathogen interactions, it has been-frequently been found that exotics are better 50 able to escape natural enemies, possibly promoting their invasion success (Mitchell \& 51 Power, 2003; Torchin, Lafferty, Dobson, McKenzie, \& Kuris, 2003). For the-plant-

52 herbivore interactions, while some studies have shown that exotic plant species incurred less herbivore damage than natives (Agrawal et al., 2005; Cappuccino \& Carpenter, 2005; Carpenter \& Cappuccino, 2005; Jogesh, Carpenter, \& Cappuccino, 2008; Liu, Stiling, \& Pemberton, 2007), others have revealed that exotic plants suffered more damage than natives (Agrawal \& Kotanen, 2003; Heard \& Sax, 2013; Morrison \& Hay,

57 2011; Parker \& Hay, 2005), or that there was no difference in herbivore damage between exotic and native plants (Chun, Van Kleunen, \& Dawson, 2010; Meijer, 
Schilthuizen, Beukeboom, \& Smit, 2016). There are several potential explanations for

60 these discrepancies in the scientific literature. Firstly, enemy release (ER) is evidenced

byrequires less damage not only in the introduced range of exotic species in comparison with the native range (a biogeographical comparison), but also for exotic species as compared with co-occurring native species in the invaded community (a communityscale comparison) (Colautti et al., 2004). The results from these two types of comparisons may be different. For example, Hawkes (2007) showed that invasive plants in their introduced range suffered lower herbivore damage than in their home native range, whereas in the invaded community ${ }_{2}$ invasive plants and native congeners did not differ in herbivore damage. Furthermore, for most studies, the number of species used were relatively small and commonly focused on specific and narrow taxonomic groupsa. As for the meta-analyses on the subject, the results were commonly derived

71 from a very limited number of studies. Due to these small numbers of species, the effect

72 of ER was probably obscured by variance arising from species characteristics and the local environment. Finally, contrasting results may be obtained if different measures of herbivore damage are adopted. Studies adopting herbivore diversity or abundance as a substitute for herbivore damage frequently reached different conclusions from those using actual tissue loss due to herbivory (Liu \& Stiling, 2006; Meijer et al., 2016). In order to better assess whether exotic plants pervasively consistently incur less herbivory pressure than natives, it may be necessary to use a large number of species, to measure their actual herbivore-induced damage, and to conduct the comparisons at both the biogeographical and community levels. presumed differential herbivory latitudinal gradient (HLG) between exotic and native plant species (Bezemer, Harvey, \& Cronin, 2014). Bezemer et al. (2014) suggested that 
native plants usually showed a decreasing herbivory rate with latitude, possibly based on a co-evolution between the native plants and herbivores for a significant amount of time; whereas, exotic plants might not have enough time to develop a co-adaptation to native herbivores, and thus may not evolve a latitudinal gradient in plant defense or palatability, like native plants de. This difference in HLG between exotic and native plants probably may results in a-decreasing ER strength with latitude (Figure S1 A), helping to explain the inconsistent results on the ERH mentioned above. This largescale heterogeneity in relative herbivory pressure on exotic and native plants, if translating to performance advantage, may also help to understand why exotic species invade successfully in some geographical locations but not in others (Allen et al., 2017; Bezemer et al., 2014; Cronin, Bhattarai, Allen, \& Meyerson, 2015). Cronin et al. (2015) provided observational evidence for this decreasing ER with latitude, finding that chewing leaf damage and stem-feeder incidence decreased with latitude for natives, but not for the invasive genotype of Phragmites australis, resulting in greater herbivore pressure on natives than invasive genotypes at low latitude, but few differences at higher latitude.

Although this hypothesis seems plausible and empirically validated with a model species, some limitations still remain. On the one hand, the occurrence and direction of HLG for native species is still controversial. While lower diversity, abundance, and the specialization of herbivores at high latitudes may lead to a negative HLG, lower defenses and tolerance of plants at these latitudes likely result in an opposite pattern (Anstett, Nunes, Baskett, \& Kotanen, 2016). In practice, while many studies show higher herbivore damage at low latitudes (i.e., negative HLG) (Coley \& Barone, 1996; Lim, Fine, \& Mittelbach, 2015; Pennings et al., 2009; Schemske, Mittelbach, Cornell, Sobel, \& Roy, 2009), a positive or no relationship has also been frequently been 
reported (Moles, Bonser, Agb, Wallis, \& Foley, 2011). In a meta-analysis, Moles et al.

110 (2011) showed that only $37 \%$ of studies exhibited negative HLG, while $21 \%$ and $37 \%$

111 of studies exhibited positive and no HLG, respectively. On the other hand, some studies

112 have revealed that exotic plants could develop an HLG as well (Kambo \& Kotanen,

113 2013; Nunes, Cassin, \& Kotanen, 2016). Consequently, the HLG for both exotic and

114 native plants and the corresponding ER patterns seem to be much more complicated

115 than what was expected by Bezemer et al. (2014) (Figure S1). Until now, few studies

116 have explicitly identified and tested these potential ER patterns with latitude. Limited

117 evidence come from the invasive and native genotypes of a model species $P$. australis,

118 where an interaction effect between origin and latitude on herbivore damage, and thus varying ER with latitude, were revealed (Allen et al., 2017; Bhattarai et al., 2017; Cronin et al., 2015).

Here, by using three large herbivory datasets, we tested the ERH by comparing the-herbivory rate between exotic and native plants, and examined whether ER could variesy with latitude. Firstly, based on a leaf herbivory rate (LHR) dataset including 1,098 plant species, spanning c. 110 degrees of latitude, we compared LHR between exotic and native plant species across all taxa, between the introduced and native range of the same exotic species, and between co-occurring exotic and native and exotic plant species. Then, in order to assess any the-variations inef ER with latitude, we tested whether origin (exotic vs. native) interacts with latitude to explain the LHR variation. Further, uUsing two ER effect-size datasets, we also-conducted two meta-regressions

130 in order to verify the relationship between ER and latitude. Given that plant growth 131 forms (woody vs. non-woody) have been found to crucially affect the-herbivory rates 132 (Turcotte, Davies, Thomsen, \& Johnson, 2014; Zhang, Zhang, \& Ma, 2016), we also 
examined whether the effect of origin ${ }_{2}$ and the interaction effect between origin and latitude ${ }_{2}$ could changes with these different plant growth forms.

\section{METHODS}

\subsection{Data acquisition and compilation}

We used a global herbivory dataset compiled by Turcotte et al. (2014), which includes the annual or daily lteaf hHerbivory rRate (LHR; - (percentage of leaf area consumed) across 1,145 species of vascular plants collected from 189 studies. Given that the vast majority of data at daily resolution are is absent in this dataset, we only used the annual LHR involving 1,021 species and 165 studies. We searched each paper associated with this dataset in order to determine whether plant species were identified as exotic or native or exotic for each specific study site. We further searched the DAISIE (www.europe-aliens.org) and USDA (http://plants.usda.gov) databases in order to recheck species' geographical origin (exotic vs. native). Additionally, we searched the Web of Science and Google Scholar using the keywords "herbivory", "enemy release", "latitude" and "herbivore-plant interactions", and identified another 16 studies containing data for 123 plant species (of which, 46 species also occurred in Turcotte's dataset). These studies were chosen according to the following criteria: (1) only HLHR representing an annual mean herbivory area percentage was included, while daily herbivory rates and other plant tissue herbivory rates were excluded; (2) experimental studies focusing on only one specific herbivore were excluded; (3) herbivore damage calculated by frequency, score, and specific index were excluded; and (4) studies that did not provide the herbivory rate of specific plant species were excluded. For each species in these studies, we extracted the mean annual herbivory rate from figures using the R package metaDigitise (Pick, Nakagawa, \& Noble, 2019), 
and recorded the location, geographic origin, and growth form (woody or non-woody). Then, we combined these herbivory data with Turcotte's dataset (Turcotte et al., 2014), producing a combined dataset that included 1,098 species (955 native and 175 exotic species; 677 woody and 421 non-woody plants) from 460 study sites spanning c. 110 degrees latitude (from $68.0^{\circ} \mathrm{N}$ to $41.6^{\circ} \mathrm{S}$ ) and almost all biomes (Figure 1). Using this global leaf herbivory dataset, we compared the mean LHR and examined the latitudinal gradient in LHR for both exotic and native plants. We further extracted two subsets from the global dataset, given that the ERH predicts two specific aspects, i.e., that an exotic species will suffer lower damage in the introduced than in the home range, escaping their natural enemies, and that exotic species should suffer lower damage than coexisting native plant species in the community, giving them a competitive advantage (Colautti et al., 2004). Specifically, we identified 32 exotic species (from 31 studies and 99 study sites) occurring in both their introduced and home ranges in order to test the former aspect (corresponding to Colautti's definition, hereafter called biogeographical subset), and identified 40 study sites (from 33 studies including 328 species) to test the latter aspect, where exotic and native species co-occurred in one study site (corresponding to Colautti's definition, hereafter called community subset).

The "study site" here refers to the geographic location- where the observation was performed and where data wereas obtained. It is possible that different studies have been conducted at the same site, and that a study included a few different sites. For the community subset, while most of the co-occurring exotic and native species in one site come from one study (34 out of 40), the data for co-occurring species comes from different studies at the remaining six sites. We also re-analyzed the community subset only using the 34 sites, and found that the result was not different from that using all the 40 sites. 
In order to further verify whether ER effects changes with latitude, we compiled another two ER effect-size datasets from two independent studies (Chun et al., 2010; Meijer et al., 2016). These two studies used meta-analysis to examine the ERH, and to our knowledge, they represent a relatively complete data collection concerning the ERH in terms of herbivore damage. Chun et al. (2010) calculated 109 ER effect sizes (log response ratio of exotic to native plants in enemy damage: disease prevalence, herbivore richness, leaf damage, or seedling damage) and associated variance for 72 exotic-native_-exotic species pairs from 19 studies. We obtained the geographical coordinates from either the original study or the online geographic tool called "LatLong" (https://www.latlong.net). Meijer et al. (2016) calculated 49 ER effect sizes for cooccurring exotic and native plants from 32 studies (five studies are-overlapped with those used by Chun et al. (2010)), as well as 19 ER effect sizes for exotic species occurring both in their introduced and home ranges from 15 studies (Hedges' $d$ as a metric of ER in enemy damage: insect abundance, insect richness, or leaf damage). We excluded the 19 ER effects for exotic versus the native range because these studies were conducted at two geographical locations, and the calculated effect was unable to be matched to a unique latitude coordinate. In order to make the effect sizes comparable to those from Chun et al. (2010), we re-calculated the log response ratio and associated variance for the remaining 49 ER effect sizes using the escalc() function in the metafor package (Viechtbauer, 2010). Finally, we obtained the geographical coordinates using the approach discussed above. Due to the different data structures for these two datasets, we re-analyzed them separately as well.

\subsection{Data analysis}

We performed all statistical analyses using R 3.6.0 (R Core Team, 2019). Prior to data analysis, the herbivory rate was logit-transformed in order to improve distribution 
normality, as suggested by Warton and Hui (2011), and the homogeneity of variances was checked with the levenTest() function in the car package (Fox et al., 2012). Using herbivory datasets, Bayesian mixed models were performed in order to test the effect of origin and its interactions with both latitude and growth forms, while also taking into account the phylogenetic correlation and spatial autocorrelation. These Bayesian mixed models were implemented in the INLA package, which used integrated nested Laplace approximation for Bayesian inference (Rue, Sara, \& Nicolas, 2009). Using the ER effect-size datasets, two inverse-variance-weighted hierarchical meta-regressions were performed in order to directly examine the relationship between ER effect and latitude, while also considering the non-independence of multiple effects within one study. Both meta-regressions were implemented using the metafor package (Viechtbauer, 2010).

\subsubsection{Constructing phylogenetic and spatial variance-covariance matrixes}

We constructed a phylogenetic tree with branch length for 1,098 species using the V.PhyloMaker package (Jin \& Qian, 2019), based on a combination of the Zanne et al. (Zanne et al., 2014) and Smith and Brown (Smith \& Brown, 2018) plant phylogenies (Figure S2). A significant phylogenetic signal was detected for herbivory rate among species with the phytools package (Revell, 2012) (Pagel's $\lambda=0.678, P<0.001$ ). In order to account for the phylogenetic correlation, we obtained the phylogenetic variance-covariance matrix from the tree using the ape package (Emmanuel, Julien, \& Korbinian, 2004). A significant spatial autocorrelation among herbivory rates was also detected with the ape package (Moran'I $=0.317, P<0.001$ ), and to account for this, we also constructed a spatial variance-covariance matrix among study sites using the method provided by Kubelka et al. (2018). The details for the construction of the phylogenetic and spatial covariance matrixes are available in the supporting information in Section 2. 


\section{in herbivory}

In order to evaluate whether the effects of origin (exotic vs. native) and latitude on herbivory were different between the Northern and Southern hemispheres, we tested the interaction effect between origin and hemisphere, as well as the interaction effect between latitude and hemisphere. Since these interactions were not significant (supporting information, Section 4, Figure S6 and S7), we used the absolute value of latitude in all of the following models (see Chen et al., 2019). Additionally, a previous study showed that a unimodal rather than a linear herbivory latitudinal pattern could possibly occur (Kozlov, Lanta, Zverev, \& Zvereva, 2015). In order to evaluate this nonlinear pattern, we also fitted models including the quadratic term (latitude ${ }^{2}$ ) (also see Allen et al., 2017) and compared them to linear models based on the DIC (deviance information criterion) value (Wang, Ryan, \& Faraway, 2018). The models including the quadratic term were not superior to linear models (supporting information, Section 5), so we used the linear fittings in all of the following models.

\subsubsection{Testing the enemy release hypothesis $\underline{(\mathrm{ERH})}$}

In order to compare native and exotic and native species' herbivory rates, we conducted our analyses separately for: (i) the full dataset; (ii) the biogeographical subset (i.e., comparing the herbivory rate of exotic species only between their introduced and native ranges); and (iii) and-the community subset (i.e., comparing the herbivory rate of exotic species against co-occurring native species). For the full dataset, origin (exotic vs. native) was used as a unique fixed effect, with species and study site as random effects $_{2}$ in order to account for variations in $\ominus$ f the herbivory rate among species and study sites. To account for phylogenetic correlations among species and spatial 

matrixes constructed above were added through the $f()$ function in INLA. For the biogeographical and community subsets, the same fixed and random effects were used, while the variance-covariance matrixes were extracted from the global matrixes above based on the specific species and study sites.

\subsubsection{Testing the interactive herbivory latitudinal gradient}

Prior to testing the interaction effect between origin (exotic vs. native) and latitude, we also examined the overall herbivory latitudinal gradient (i.e., testing the latitudinal herbivory hypothesis) by including latitude as a unique fixed effect. In order to test whether exotic and native plants differ in their herbivory latitudinal gradient, we used origin, latitude, and their interaction term as fixed effects to fit a full model, and then examined the significance of the interaction effect. We tested the interaction effect for each dataset separately: (i) the full herbivory dataset; (ii) the biogeographical subset; and (iii) the community subset. Then, a reduced model without the interaction term was fitted and compared with the full model based on the $D I C$ value in order to check the significance of the interaction effect. We then quantified the latitudinal gradient for both the exotic and the native species, respectively. For all the models, the species and study sites were used as random effects with phylogenetic and spatial variancecovariance matrixes included in order to account for phylogenetic and spatial autocorrelation. Since climatic conditions, e.g., annual mean temperature, could be largely different at the same latitude on different continents, these differences may obscure the signal of the herbivory gradient obtained from latitude. We further checked whether the herbivory gradients and its interaction with origin still held using the annual mean temperature of each geographical location to replace the-latitude for the full herbivory dataset. The annual mean temperature at the finest 30 arcsec spatial 
resolution was obtained from the WorldClim version 2.1 (https://www.worldclim.org) with the package raster (Hijmans et al., 2020).

In order to evaluate the influence of growth form (woody vs. non-woody), we examined the interaction effect between origin and growth form, as well as between latitude and growth form. We also quantified the effects of origin and latitude, along with their interactions for woody and non-woody plants, respectively. For all models, species and studies were used as random effects with phylogenetic and spatial variancecovariance matrixes being added. The posterior distributions of the fixed effects were reported for all of the analyses above, where 95\%_CI (credible interval) excluding 0 indicates a significant effect and including 0 indicates a non-significant effect.

In order to verify whether additional covariance matrixes have dealt with the phylogenetic and spatial autocorrelation, we also checked the phylogenetic signal and spatial autocorrelation in the residuals of all our models. We found that the phylogenetic signal and spatial autocorrelation had been removed to a great extent (supporting information, Section 3, Figure S3, S4 and S5).

\subsubsection{Meta-regression}

In order to further verify the relationship between ER and latitude, we performed two meta-regressions using the rma.mv() function in the metafor package. For the Chun et al. (2010) datasets, the effects of ER (log response ratio) were used as a function of absolute latitude, with the inverse variance of effect as a weight. Study and species pairs were used as random effects in order to statistically account for the non-independence of multiple effects within one study, and of one species pair used for multiple studies. For the Meijer (2016) datasets, the inverse-variance weighted regression between Commented [A6]: et al?

304 effects and absolute latitude was also used, but with study as the only random effect, because the species pairs were not identified in this dataset. 


\subsection{Comparison of herbivory rate between exotic and native species}

Across all species, the mean herbivory rate for exotic plants was significantly lower than that of the natives (Figures 2A and 3; Table S1). When considering the specific biogeographical and community datasets, the herbivory rate was lower not only for exotic species in their introduced range compared to than in their home range (Figures 2B and 3; Table S1), but also for exotic species compared tothan the natives co-occurring in the same community (Figures $2 \mathrm{C}$ and 3; Table $\mathrm{S} 1$ ). We found a significant interaction effect between growth form and origin (Table S3), indicating that the effect of origin on herbivory rate (i.e., ER) changed with plant growth form. Specifically, woody exotic species had significantly lower herbivory rates than natives, but there was no significant difference for non-woody plants (Figures 3 and 4; Tables S4 and S5).

\subsection{The interaction effect between origin and latitude}

HThe herbivory rate did not change significantly with increasing latitude (Figure 3; Tables S1 and S2). Across all species, no significant interaction effect between origin and latitude was found (Figures 2A and 3; Table S1). The model comparison also indicated that the model including the interaction effect $(\mathrm{DIC}=4544.05)$ was no better than models with the additive effects of origin and latitude $(\mathrm{DIC}=4542.65)($ Table S1). Similarly, there was no significant interaction effect between origin and latitude for exotic species when comparing the-herbivory rate between the introduced range and the home range (Figures $2 \mathrm{~B}$ and 3; Table $\mathrm{S} 1$ ), nor when comparing the-herbivory rate between exotic and native species co-occurring in the same community (Figures 2C

329 and 3; Table S1). Even although we distinguished between plant growth forms, the 

non-woody plants (Figures 3 and 4; Table S4). A similar pattern was also found when using temperature as a proxy for latitude, where herbivore damage did not change significantly with increasing temperature across all species, and there was no significant interaction effect between origin and temperature (Figure S8; Table S6).

\subsection{The relationship between enemy release and latitude}

From our two independent meta-regressions, we verified that ER effects did not change with latitude. Whether using meta-regression with species pairs characterizing effects (Figure 5A), or with studies characterizing the effects (Figure 5B), there was no significant relationship between ER and latitude.

\section{DISCUSSION}

Using three large global herbivory datasets, we compared leaf herbivory ratethe (LHR) between exotic and native plant species and investigated the-variation in enemy $\underline{\text { release }} \theta f(E R)$ effects across $w i t h$ plant growth forms and latitudinal gradients. We found a-widespread ER in the LHR, and revealed a contrasting ER pattern between woody and non-woody plants. We also found similar patterns of herbivory across a latitudinal gradient between exotic and native species (i.e., no interaction effect between species origin and latitude on herbivory), thus indicating that ER does not change with latitude, which was further verified by two meta-regressions. Our findings may provide some insights into understanding and predicting biological invasions.

\subsection{Testing the enemy release hypothesis}

We found that exotic plant species suffered $13 \%$ less leaf herbivore damages than 352 natives. Our study used the largest number of species to date (175 exotic and 955 native 
and 175 exotic species), considered two levels (biogeography and community) of the enemy release hypothesis ( $\mathrm{ERH}_{2}$ (Colautti et al., 2004), and statistically accounted for phylogenetic and spatial autocorrelation. One potential reason for the lack of consensus on the ERH across studies is the often small sample size and restricted spatial extent used in case studies, as well as limited numbers of studies used in meta-analyses. While herbivore damage could vary among plant species (Agrawal et al., 2005), natural enemies (Parker \& Gilbert, 2007) and habitat types (Knapp, Fownes, \& Harrington, 2008), any general ER effect could be masked by different biotic/abiotic environments. Therefore, different studies using different species in different habitats may lead to contrasting conclusions. From our results (Figure 2), we can see that for specific species and at specific geographic locations, exotic plants probably-may suffer similar, higher, or lower herbivore damage than natives. Thus, small samples weaken theeur ability to obtain the broad generality of ER, and similarly, the small number of studies used in the previous meta-analysis may also lower the statistical power to detect significant LHR differences between exotic and native species.

When focusing only on exotic species and comparing the-herbivory rate between the native and introduced range, we revealed that plant species in their introduced range experienced $37 \%$ less leaf herbivore damage than those in their native range (Figure 2B). When comparing the herbivory rate between exotic and native species cooccurring in the same community, we revealed that exotics suffered $8 \%$ less leaf herbivore damage than co-occurring native species (Figure 2C). As noted by Colautti et al. (2004), even although release from natural enemies occurred in the introduced range, exotic species might not obtain an advantage over co-occurring natives if the latter suffer similar low damage by enemies. Different or opposite results are commonly obtained for these two comparisons (Colautti et al., 2004). For example, Hawkes (2007) 
found significant differencesresults for biogeographic comparisons, but not for community-level comparisons, while the opposite was reported by Liu \& Stiling (2006). Here, our results support the ERH in both comparisonssituations. We demonstrated that exotic plants are not only better able to escape enemies in the introduced range, but they also incur less herbivore damage than co-occurring natives. Thus, if this ER translates to better performance, exotic plants are likely to obtain a competitive advantage, potentially promoting their invasion.

There is growing appreciation that species- traits have a phylogenetically related structure, which violates the precondition of statistical independence when conducting a multiple-species study (Ives \& Helmus, 2011; Webb, Ackerly, McPeek, \& Donoghue, 2002). We found a significant phylogenetic signal for species herbivory rates. In order to solve this problem, a common method is to conduct the phylogenetic generalized least square regression (PGLS) (Martins \& Hansen, 1997). However, this technique is unable to simultaneously include random effects, which is also necessary for most survey studies to account for the variance among species and study sites. Alternatively, spatial autocorrelation usually exists for multiple-site studies, but is commonly ignored in most studies (Koenig, 1999). Using the Bayesian framework, we explicitly included phylogenetic and spatial structures in our mixed models, and verified that these including matrixes indeed improved these issues to a great extent. These statistical approaches can thus make our results more conservative compared to previous studies. Even so, we found evidence that the-herbivore damage for exotic plants was pervasively less than that for

However, it should be noted that we did not examine the link between ER and plant performance. It has been pointed out that herbivore damage does not necessarily translate to plant performance, given that different species may have different 

do not know whether and to what extent the lower herbivore damage on exotic plants translates $\mathrm{e}$ to performance advantages and increased population growth over that of natives, which is required in order to fully assess the ERH (Chun et al., 2010; Keane \& Crawley, 2002). It should also be noted that our study only included LHR, and it is possible that other herbivory damages, such as floral herbivory or seed predation, may have different ER effects. In order to fully evaluate the ERH, other herbivory damages should be considered in future studies. Overall, our results indicate that ER is pervasive in terms of leaf herbivory rates, and the next challenge is likely to try to understand what this widespread lower herbivory pressure means for the performance and invasion success of exotic species.

\subsection{Contrasting enemy release for woody and non-woody plant species}

We found that ourthis result of lower herbivory rate in exotic than native plant species was mostly driven by woody plants, regardless of using all species, comparing the exotic and native range, or comparing co-occurring exotic and native species in the same community (Figures 3 and 4; Table S4, S5). To our knowledge, few studies have compared ER between woody and non-woody plants, which may also be a reason why there are inconsistent results in the scientific literatures on testing the ERH. Through identifying the origin (exotics or natives) of woody and non-woody plants in our herbivory database, we revealed that ER was only significant for woody plant species, but not for non-woody species. Woody plants tend to have larger and more apparent leaves, as well as longer leaf life spans, possibly increasing the chance ofte suffering from herbivore damage (Feeny, 1976; Turcotte et al., 2014; Zhang et al., 2016). In addition, longer life spans also possibly indicate that they accumulate damages over 
time (Southwood, Brown, \& Reader, 1986). It is also possible that woody and nonwoody plants have discrepancies in defense or palatability to herbivores, resulting in a corresponding difference in ER. Turcotte et al. (2014) found that woody plant species could experience a $64 \%$ higher LHR than non-woody plants. This higher herbivory rate for native woody plants could lead to stronger ER for woody plants. However, it should also be noted that the bigger and longer-lived leaves may increase the chance of detecting the herbivore damage by investigators, potentially leading to a discrepancy in the observed damage between woody and non-woody plants. Overall, our findings suggest that plant growth forms have a strong influence on examining the ERH, and more studies are required in order to test the causes underlying this discrepancy between woody and non-woody plants.

\subsection{Latitudinal gradient in herbivore damage}

The relationship between herbivory damage and latitude has been evaluated by a number of studies (Moles et al., 2011). However, to date, there does not appear to be a clear consensus. Some potential issues on testing the herbivory latitudinal gradient (HLG) hypothesis have been recently demonstrated (Anstett et al., 2016) and, in particular, phylogenetic and spatial scales were identified as important limiting factors.

We explicitly considered phylogenetic and spatial autocorrelations in the analyses (supporting information, Section1). We did not find a significant variation in LHR with latitude, regardless of using the full dataset, biogeographical subset, community subset, or distinguishing between plant growth forms (Figure 3; Tables S1 and S4). We also found a similar result when using temperature instead of latitude (supporting information, Section 7). Our result was inconsistent with one recent study (Lim et al., 2015), which showed a significant decrease in LHR with latitude, as well as an apparent difference between woody and non-woody plants. Compared to this study, a main 
difference in our_study is that we included a spatial covariance matrix in order to statistically account for the spatial autocorrelation among the study sites. We suggest that the-violations of statistical independence should be considered in future survey studies concerning multiple spatial locations and species. Additionally, similar to most other studies (Kozlov et al., 2015; Lim et al., 2015; Zhang et al., 2016), the explanatory power of latitude in our study is low with large herbivory variation unexplained. We suggest that, compared to identifying the relationship between herbivory rate and latitude, more efforts should be devoted to identifying the key factors and assessing their relative importance in explaining herbivory variation.

It should be noted that the vast majority of species used in this study did not have a latitudinal range (from $68.0^{\circ} \mathrm{N}$ to $41.6^{\circ} \mathrm{S}$ ), i.e., our results were obtained by combining species at different locations. Thus, species identity may somewhat obscure our results.

This is a common limitation to studies using multiple species to analyze herbivory latitudinal patterns (Anstett et al., 2016; Kozlov et al., 2015; Lim et al., 2015). We also could not test the effect of the types of herbivore damages due to data limitations, although different herbivores may induce different herbivory latitudinal patterns (Anstett, Naujokaitis-Lewis, \& Johnson, 2014; Nunes et al., 2016).

\subsection{Testing the-variation in enemy release with latitude}

Based on a hypothesized different HLG between exotic and native plants, it has been proposed that ER should vary with latitude (Bezemer et al., 2014; Cronin et al., 2015). We showed that there was no interaction effect between species origin (exotic vs. native) and latitude to explain the herbivory rate, indicating that ER does not change with latitude. This inference was further confirmed by two meta-regressions, where no apparent relationships were found between ER and latitude. Our results contrast with 
previous case studies that found an interaction effect between latitude and origin on herbivory rate, and showed a-varying ER with latitude (Allen et al., 2017; Bhattarai et al., 2017; Cronin et al., 2015). These studies, while offering a mechanistic insight into HLG, may not yield generalized conclusions because they focused on a model species, P. australis, with a relatively narrow latitudinal range. Compared with these intraspecific studies, our study included a larger number of species (1,098 species) and spanned a much wider spatial scale (110 degrees global latitude). This gives our conclusions greater generality in the HLG, differences between exotic and native species, and expected latitudinal patterns in ER. We found no evidence to support the interaction effect between species origin and latitude, regardless of using all taxa, comparing the herbivory rate between the invaded and native range of exotic species, comparing the herbivory rate between exotic and native species co-occurring in the same community, or distinguishing between woody and non-woody species. Based on current evidence, we suggest that a latitudinal gradient in ER might exist for specific taxa, but it should not be extrapolated as a widespread pattern.

Our results caution against the validity of using varying ER with latitude to explain the inconsistent results on testing the ERH and to understand the geographical variation of invasion success (Allen et al., 2017; Bezemer et al., 2014; Bhattarai et al., 2017; Cronin et al., 2015). However, we suggest that deducing ER variation from a differential HLG between exotic and native plant species provides an important insight into evaluating large-scale heterogeneity in ER (Figure S1). Integrating more ecological and evolutionary factors into this framework in the future may provide a novel perspective through which to understand the spatial heterogeneity of invasions success.

\subsection{Conclusion}


In summary, our study reveals that LHR is prevalently lower for exotic plant species than it is for natives. This herbivory rate difference does not change with the latitudinal locations, implying that ER should play a similar role at both high and low latitudes. However, this ER is only obvious for woody plants, rather than for nonwoody plants. One future challenge is to possibly assess whether and to what extent this ER in woody exotic plants translates to performance advantage and influences their invasion success.

\section{DATA ACCESSIBILITY STATEMENT}

The data that support the findings of this study will be openly available in Dryad (http://datadryad.org) if the paper is accepted for publication.

\section{REFERENCES}

Agrawal, A. A., \& Kotanen, P. M. (2003). Herbivores and the success of exotic plants: a phylogenetically controlled experiment. Ecology Letters, 6, 712-715.

Agrawal, A. A., Kotanen, P. M., Mitchell, C. E., Power, A. G., Godsoe, W., \& Klironomos, J. (2005). Enemy release? An experiment with congeneric plant pairs and diverse above-and belowground enemies. Ecology, 86, 2979-2989.

Allen, W. J., Meyerson, L. A., Cummings, D., Anderson, J., Bhattarai, G. P., \& Cronin, J. T. (2017). Biogeography of a plant invasion: drivers of latitudinal variation in enemy release. Global Ecology and Biogeography, 26, 435-446.

Anstett, D. N., Naujokaitis-Lewis, I., \& Johnson, M. T. (2014). Latitudinal gradients in herbivory on Oenothera biennis vary according to herbivore guild and specialization. 
Anstett, D. N., Nunes, K. A., Baskett, C., \& Kotanen, P. M. (2016). Sources of Controversy Surrounding Latitudinal Patterns in Herbivory and Defense. Trends in Ecology \& Evolution, 31, 789-802.

Bezemer, T. M., Harvey, J. A., \& Cronin, J. T. (2014). Response of native insect communities to invasive plants. Annual Review of Entomology, 59, 119-141.

Bhattarai, G. P., Meyerson, L. A., Anderson, J., Cummings, D., Allen, W. J., \& Cronin, latitudinal clines for traits related to herbivory. Ecological Monographs, 87, 57-75. than non-invasive exotic plants. Biology Letters, 1, 435-438.

Carpenter, D., \& Cappuccino, N. (2005). Herbivory, time since introduction and the invasiveness of exotic plants. Journal of Ecology, 93, 315-321.

Chen, S. C., Tamme, R., Thomson, F. J., Moles, A. T., \& Nathan, R. (2019). Seeds tend to disperse further in the tropics. Ecology Letters, 22, 954-961.

Chun, Y. J., Van Kleunen, M., \& Dawson, W. (2010). The role of enemy release, tolerance and resistance in plant invasions: linking damage to performance. Ecology Letters, 13, 937-946.

Colautti, R. I., Ricciardi, A., Grigorovich, I. A., \& MacIsaac, H. J. (2004). Is invasion success explained by the enemy release hypothesis? Ecology Letters, 7, 721-733.

Coley, P. D., \& Barone, J. A. (1996). herbivory and plant defenses in tropical forests. 
Cronin, J. T., Bhattarai, G. P., Allen, W. J., \& Meyerson, L. A. (2015). Biogeography of a plant invasion: plant-herbivore interactions. Ecology, 96, 1115-1127. Chapman and Hall.

Emmanuel, P., Julien, C., \& Korbinian, S. (2004). APE: Analyses of Phylogenetics and Evolution in R language. Bioinformatics, 20, 289-290.

Feeny, P. (1976). Plant apparency and chemical defense. In Biochemical interaction between plants and insects (pp. 1-40): Springer.

Fox, J., Weisberg, S., Adler, D., Bates, D., Baud-Bovy, G., Ellison, S., . . Graves, S. (2012). Package 'car'. Vienna: R Foundation for Statistical Computing.

Hajek, A. E., \& Eilenberg, J. (2018). Natural enemies: an introduction to biological control (2nd ed.): Cambridge University Press.

Hawkes, C. V. (2007). Are invaders moving targets? The generality and persistence of advantages in size, reproduction, and enemy release in invasive plant species with time since introduction. The American Naturalist, 170, 832-843.

Heard, M. J., \& Sax, D. F. (2013). Coexistence between native and exotic species is facilitated by asymmetries in competitive ability and susceptibility to herbivores. Ecology Letters, 16, 206-213.

Hijmans, R. J., Etten, J. v., Sumner, M., Cheng, J., Bevan, A., Bivand, R., \& Wueest, 

analyses of community structure. Ecological Monographs, 81, 511-525.

Jin, Y., \& Qian, H. (2019). V. PhyloMaker: an R package that can generate very large phylogenies for vascular plants. Ecography, 42, 1353-1359.

Jogesh, T., Carpenter, D., \& Cappuccino, N. (2008). Herbivory on invasive exotic plants and their non-invasive relatives. Biological Invasions, 10, 797-804.

Kambo, D., \& Kotanen, P. M. (2013). Latitudinal trends in herbivory and performance of an invasive species, common burdock (Arctium minus). Biological Invasions, 16, $101-112$.

Keane, R. M., \& Crawley, M. J. (2002). Exotic plant invasions and the enemy release hypothesis. Trends in Ecology \& Evolution, 17, 164-170.

Knapp, L. B., Fownes, J. H., \& Harrington, R. A. (2008). Variable effects of large mammal herbivory on three non-native versus three native woody plants. Forest ecology and management, 255, 92-98.

Koenig, W. D. (1999). Spatial autocorrelation of ecological phenomena. Trends in Ecology \& Evolution, 14, 22-26.

Kolar, C. S., \& Lodge, D. M. (2001). Progress in invasion biology: predicting invaders. Trends in Ecology \& Evolution, 16, 199-204.

Kozlov, M. V., Lanta, V., Zverev, V., \& Zvereva, E. L. (2015). Global patterns in background losses of woody plant foliage to insects. Global Ecology and Biogeography, 24, 1126-1135. 
Kubelka, V., Šálek, M., Tomkovich, P., Végvári, Z., Freckleton, R. P., \& Székely, T. (2018). Global pattern of nest predation is disrupted by climate change in shorebirds. Science, 362, 680-683.

Lim, J. Y., Fine, P. V. A., \& Mittelbach, G. G. (2015). Assessing the latitudinal gradient in herbivory. Global Ecology and Biogeography, 24, 1106-1112.

Liu, H., \& Stiling, P. (2006). Testing the enemy release hypothesis: a review and metaanalysis. Biological Invasions, 8, 1535-1545.

Liu, H., Stiling, P., \& Pemberton, R. W. (2007). Does enemy release matter for invasive plants? Evidence from a comparison of insect herbivore damage among invasive, non-invasive and native congeners. Biological Invasions, 9, 773-781.

Mack, R. N., Simberloff, D., Mark Lonsdale, W., Evans, H., Clout, M., \& Bazzaz, F. A. (2000). Biotic invasions: causes, epidemiology, global consequences, and control. Ecological Applications, 10, 689-710.

Maron, J. L., \& Vila, M. (2001). When do herbivores affect plant invasion? Evidence for the natural enemies and biotic resistance hypotheses. Oikos, 95, 361-373. General Approach to Incorporating Phylogenetic Information into the Analysis of Interspecific Data. American Naturalist, 149, 646-667.

604 Meijer, K., Schilthuizen, M., Beukeboom, L., \& Smit, C. (2016). A review and metaanalysis of the enemy release hypothesis in plant-herbivorous insect systems. PeerJ, 4, e2778. 

pathogens. Nature, 421, 625-627. evidence for latitudinal gradients in plant defence and herbivory. Functional Ecology, 25, 380-388.

Morrison, W. E., \& Hay, M. E. (2011). Herbivore preference for native vs. exotic plants: generalist herbivores from multiple continents prefer exotic plants that are evolutionarily naïve. PLoS ONE, 6, e17227.

Nunes, K. A., Cassin, C. M., \& Kotanen, P. M. (2016). Variation in herbivory along a latitudinal gradient for native and exotic Asteraceae. Plant Ecology, 217, 481-493.

Parker, I. M., \& Gilbert, G. S. (2007). When there is no escape: the effects of natural enemies on native, invasive, and noninvasive plants. Ecology, 88, 1210-1224.

Parker, J. D., \& Hay, M. E. (2005). Biotic resistance to plant invasions? Native herbivores prefer non-native plants. Ecology Letters, 8, 959-967. marshes. Ecology, 90, 183-195.

Pick, J. L., Nakagawa, S., \& Noble, D. W. (2019). Reproducible, flexible and high throughput data extraction from primary literature: The metaDigitise $\mathrm{r}$ package. Methods in Ecology and Evolution, 10, 426-431.

627 R-Core-Team. (2019). R: A language and environment for statistical computing. R 
629 project.org

630 Revell, L. J. (2012). phytools: an R package for phylogenetic comparative biology (and 631 other things). Methods in ecology and evolution, 3, 217-223. toward understanding the ecological impacts of nonnative species. Ecological Monographs, 83, 263-282.

Rue, H., Sara, M., \& Nicolas, C. (2009). Approximate Bayesian inference for latent Gaussian models by using integrated nested Laplace approximations. Journal of the Royal Statistical Society, 71, 319-392.

Schemske, D. W., Mittelbach, G. G., Cornell, H. V., Sobel, J. M., \& Roy, K. (2009). Is There a Latitudinal Gradient in the Importance of Biotic Interactions? Annual Review of Ecology Evolution \& Systematics, 40, 245-269.

Smith, S. A., \& Brown, J. W. (2018). Constructing a broadly inclusive seed plant phylogeny. American journal of botany, 105, 302-314. expectancy and herbivore damage. Oecologia, 70, 544-548.

Torchin, M. E., Lafferty, K. D., Dobson, A. P., McKenzie, V. J., \& Kuris, A. M. (2003). Introduced species and their missing parasites. Nature, 421, 628-630. 
plants. Proceedings Biological Sciences, 281, 4739-4753.

650 Turcotte, M. M., Thomsen, C. J. M., Broadhead, G. T., Fine, P. V. A., Godfrey, R. M., 651 Lamarre, G. P. A., . . Johnson, M. T. J. (2014). Percentage leaf herbivory across vascular plant species. Ecology, 95, 788-788.

653 Viechtbauer, W. (2010). Conducting meta-analyses in R with the metafor package. Journal of Statistical Software, 36, 1-48.

Wang, X., Ryan, Y. Y., \& Faraway, J. J. (2018). Bayesian Regression Modeling with INLA: Chapman and Hall/CRC.

Warton, D. I., \& Hui, F. K. (2011). The arcsine is asinine: the analysis of proportions in ecology. Ecology, 92, 3-10.

Webb, C. O., Ackerly, D. D., McPeek, M. A., \& Donoghue, M. J. (2002). Phylogenies and community ecology. Annual review of ecology and systematics, 33, 475-505. G., .. Reich, P. B. (2014). Three keys to the radiation of angiosperms into freezing environments. Nature, 506, 89-92.

Zhang, S., Zhang, Y., \& Ma, K. (2016). Latitudinal variation in herbivory: hemispheric 

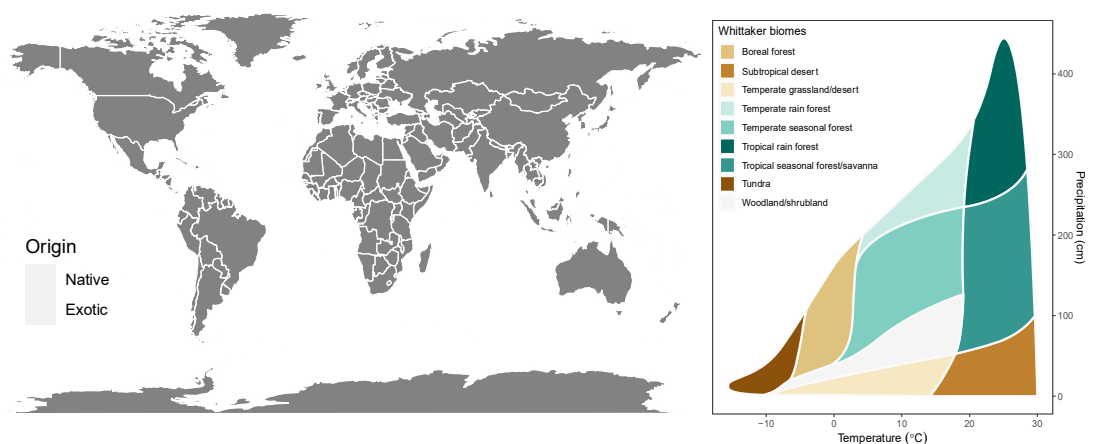

675 Figure 1. The geographical locations and the Whittaker biomes of 460 study sites for

exotic and native plant species across the globe. Circle size is proportional to the mean annual herbivory rate.

678

679

680

681

682

683

684 
687

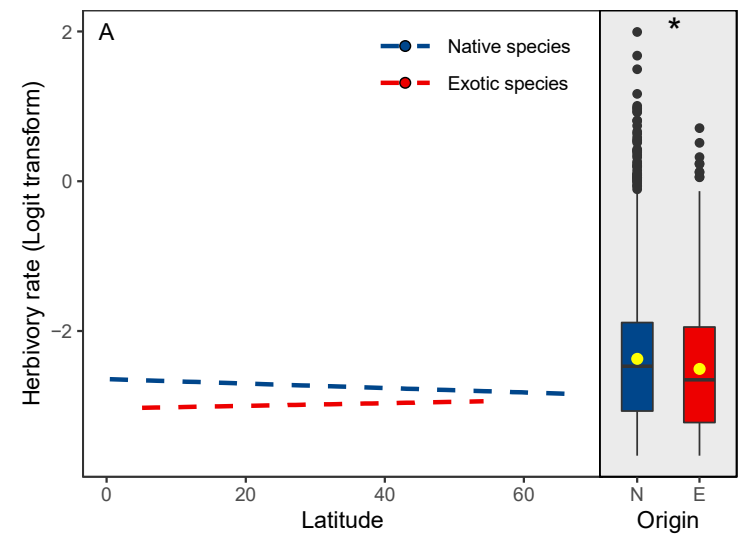

689
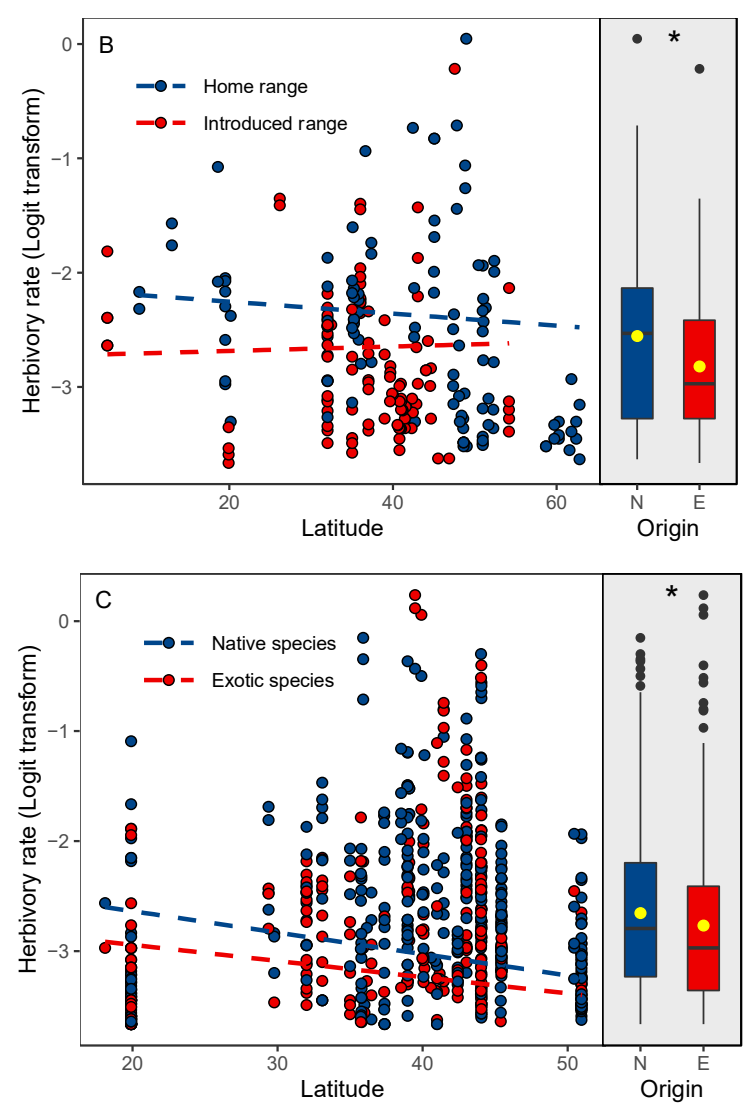

690 
691 Figure 2. Latitudinal gradients and distributions of herbivory rate (logit transform) for

692 693 the range and black points represent the outliers. Yellow points indicate the mean value exotic and native plant species across (A) all 1,098 species at the global scale, (B) species occurring inat both exotic and native ranges (biogeographical subset), and (C) co-occurring exotic and native species (community subset). Each point represents the annual mean herbivory rate of one species at one study site. Regression lines were predicted by Bayesian mixed models implemented in INLA with phylogenetic correlation and spatial autocorrelation taken into account. Dashed lines indicate that $95 \%$ CI for coefficient estimate of latitude includes 0 . Boxplots in the shaded portion show herbivory rate distributions for exotic and native plant species. The boxes show the first and third quartiles, the horizontal lines indicate the median, and vertical lines indicate and * indicates that the $95 \%$ CI of origin effect (the difference of estimated mean herbivory rate between exotics and natives) does not include 0 . 


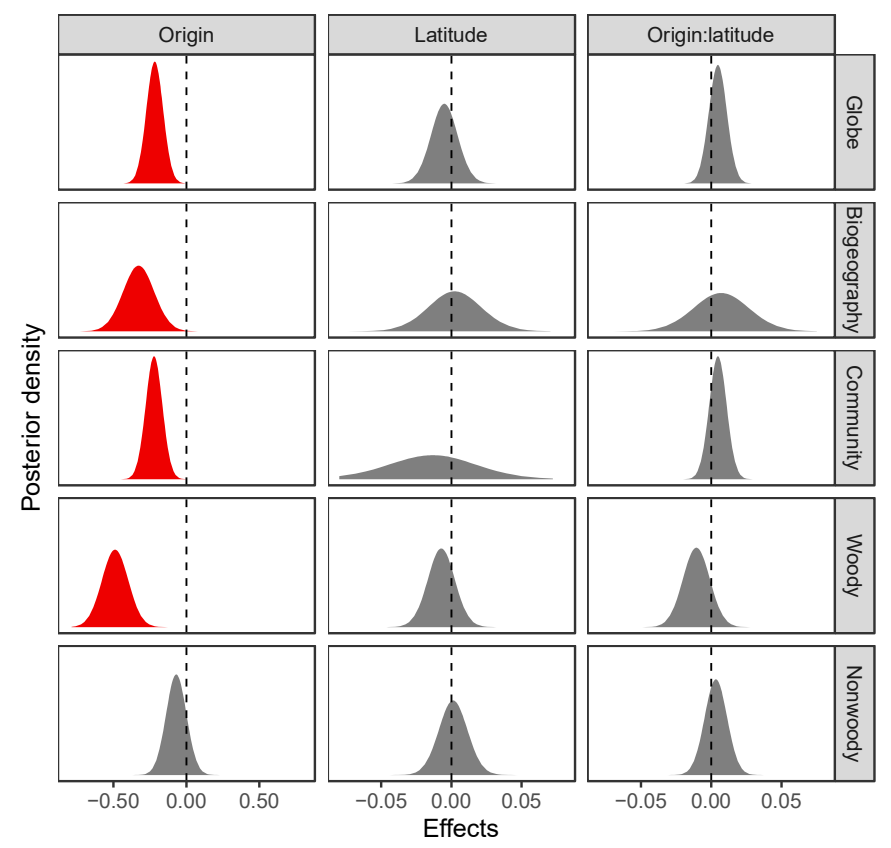

711 Figure 3. Posterior distributions for effects of origin (exotic vs. native) and latitude,

712 and their interaction effects. Posterior distributions were estimated using Bayesian

713 mixed models implemented in INLA with phylogenetic correlation and spatial

714 autocorrelation taken into account for: (1) all 1,098 species across the globe, (2) species

715 occurring inat both exotic and native ranges (biogeographical subset), (3) co-occurring exotic and native species (community subset), (4) woody plant species, and (5) non-

717 woody plant species. Origin and latitude effects were obtained with origin and latitude

718 as a unique fixed effect respectively, and interaction effects were obtained from the

719 models with origin, latitude, and origin:latitude as fixed effects. Dashed lines for effect $720=0$. Red denotes that the $95 \%$ CI of effects does not include $0_{2}$ while gray includes 0 . 


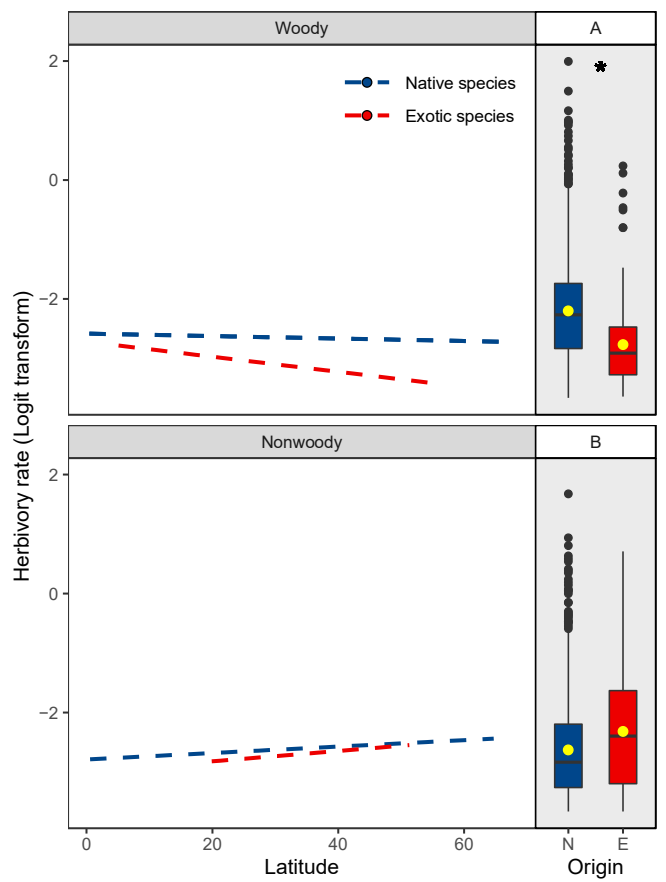

Figure 4. Latitudinal gradients and distributions of herbivory rate (logit transform) for exotic and native plant species across: (A) woody, and (B) non-woody plants.

Regression lines were predicted by Bayesian mixed models implemented in INLA with phylogenetic correlation and spatial autocorrelation taken into account. Dashed lines indicate that $95 \%$ CI for coefficient estimate of latitude includes 0 . Boxplots in the shaded portion show herbivory rate distributions for exotic and native plant species. The boxes show the first and third quartiles, the horizontal lines indicate the median, vertical lines indicate the range and black points represent the outliers. Yellow points indicate the mean value and $*$ indicates that the $95 \%$ CI of origin effect (the difference of estimated mean herbivory rate between exotics and natives) does not include 0 . 

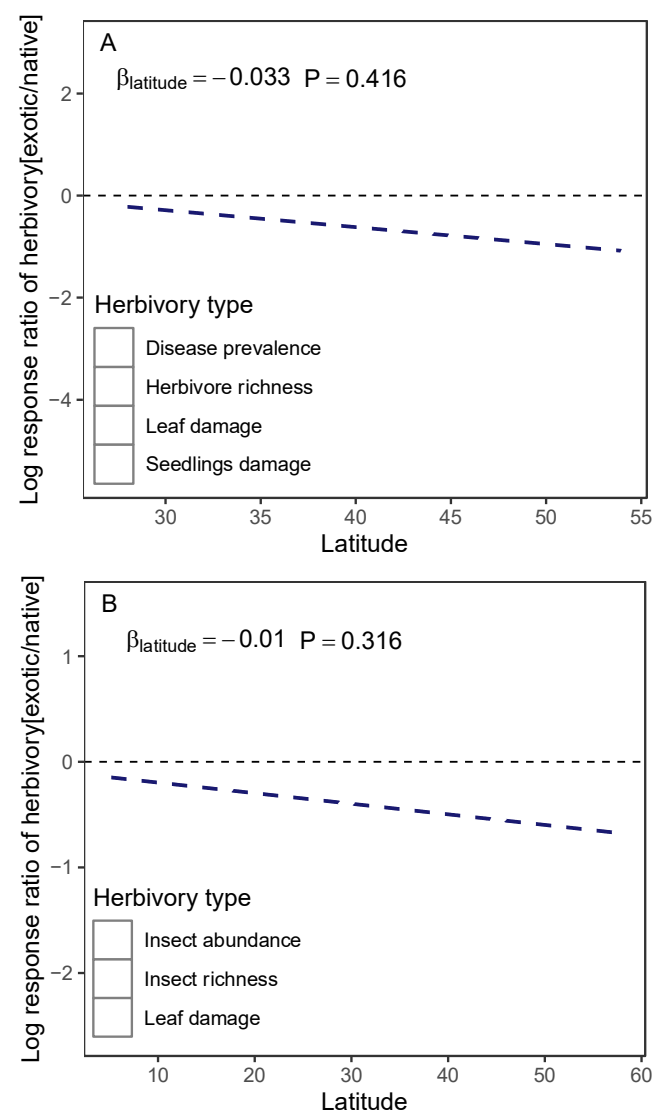

735 Figure 5. Change in strength of enemy release with latitude: (A) Meta-regression using

19 studies including 109 effect sizes for 72 species pairs; and (B) Meta-regression using

32 studies including 49 effect sizes. Dashed thin line for $\log$ response ratio $=0$; Dashed thick line for predicted mean effect size (with 95\%_CI in gray). Datapoint size is proportional to the precision (the inverse of the variance). 